\title{
Cartilage rehydration: the sliding-induced hydrodynamic triggering
} mechanism

\author{
Carmine Putignano ${ }^{a, c, *}$, David Burris ${ }^{b}$, Axel Moore ${ }^{b}$ and Daniele Dini ${ }^{c}$ \\ ${ }^{a}$ Department of Mechanics, Mathematics and Management, Politecnico di Bari, Via Orabona 4, 70100, Bari, Italy. \\ ${ }^{b}$ Department of Mechanical Engineering, University of Delaware, Newark, DE, United States of America. \\ ${ }^{c}$ Department of Mechanical Engineering, Imperial College London, South Kensington Campus, Exhibition Road, London SW7 2AZ, United Kingdom.
}

\section{ARTICLE INFO}

Keywords:

cartilage mechanics

rehydration in porous materials

multiscale porous lubrication

\begin{abstract}
A B S TRACT
Loading-induced cartilage exudation causes loss of fluid from the tissue, joint space thinning and, in a long term prospective, the insurgence of osteoarthritis. Fortunately, experiments show that joints recover interstitial fluid and thicken during articulation after static loading, thus reversing the exudation process. Here, we provide the first original theoretical explanation to this crucial phenomenon, by implementing a numerical model capable of accounting for the multiscale porous lubrication occurring in joints. We prove that sliding-induced rehydration occurs because of hydrodynamic reasons and is specifically related to a wedge effect at the contact inlet. Furthermore, numerically predicted rehydration rates are consistent with experimentally measured rates and corroborate the robustness of the model here proposed. The paper provides key information, in terms of fundamental lubrication multiscale mechanisms, to understand the rehydration of cartilage and, more generally, of any biological tissue exhibiting a significant porosity: such a theoretical framework is, thus, crucial to inform the design of new effective cartilage-mimicking biomaterials.
\end{abstract}

\section{Introduction}

For decades, articular cartilage has drawn significant research interest from an increasingly vast multi-disciplinary scientific community, which includes orthopaedists, biologists, and engineers [1][2][3]. Indeed, cartilage plays a crucial role in any articular joint, where it ensures outstanding mechanical properties in terms of high resilience, considerable load-carrying capacity and remarkably low friction [4][5][6]. Regrettably, cartilage degenerates in a diseased or osteoarthritic joint for reasons that remain unclear. Osteoarthritic degradation impairs the ability of cartilage to retain the interstitial hydration necessary for its load support and lubricating functions: this leads to gradual wear and, eventually, to failure. Osteoarthritis remains, indeed, a massive social and economic problem without a solution.

From a biomechanical point of view, the key distinguishing feature between physiological and pathological conditions is the intricate lubrication regime occurring when two cartilage interfaces are in contact and wetted by synovial fluid. While many aspects of this lubrication process remain uncertain, the lubrication success of cartilage is widely attributed to its unique biphasic structure-function relationship. Crucially, cartilage is a porous or biphasic medium comprising approximately $80 \%$ synovial fluid, a fibrous collagenous scaffold, charged molecules called glycolaminoglycans, and, finally, cells [6][7][8]. During loading, interstitial fluid is automatically pressurized to support the load and lubricate the contact hydrostatically [9], but, at the same time, the hydrostatic pressure drives fluid out from the tissue over time [10][11]. In situ measurement of articular cartilage deformation in intact femoropatellar joints under static

\footnotetext{
*Corresponding author. Phone: +390805962711 carmine. putignano@poliba. it (C. Putignano) ORCID(s): $0000-0001-6225-9630$ (C. Putignano)
}

loading have shown that the cartilage in the human knee can thin by more than $50 \%$ in just an hour or two of static loading [12]. This gradual loss of interstitial fluid defeats interstitial pressure and the associated functions of load support and lubrication. Indeed, long periods of static loading cause joint space thinning, the most common clinical diagnostic for osteoarthritis [9][10][11][13][14][15].

Fortunately, experiments show that joints recover interstitial fluid and thicken during articulation after static loading, thus reversing the exudation process and ultimately preventing excessive joint space narrowing and the irreversible damage it likely causes [16][17]. Thus, movement is the engine that sustains cartilage interstitial hydration, pressure, and lubrication over the long term. However, while it is clear that the rehydration process is critical for sustaining jointspace and, thus, health, the understanding of its underlying mechanism/s is still missing [18][24]. Indeed, while fluid exudation due to joint normal loading is quantitatively predictable with existing biphasic mechanics, the ability of cartilage to recover net fluid at the loaded contact interface due to sliding was not anticipated, even qualitatively. Crucially, such a theme not only features aspects of significant theoretical importance but has also tremendous practical implications: dehydrated cartilage means high friction, wear and, ultimately, the occurrence of degradation processes, like osteoarthritis [34]. Elucidating the mechanics of this experimentally proven rehydration process is now a priority.

Physical activity has been known to restore cartilage hydration following static loading since early experimental observations by Ingelmark and Ekholm in Ref. [25][26]. They and others have explained this phenomenon with the so-called intermittent bath exposure hypothesis: during articulation, the contact area is intermittently relieved from contact, thus enabling passive fluid recovery via osmotic swelling of the 
free dehydrated surface. The mechanisms requires that the migrating contact areas (MCAs) are periodically exposed to the bath. Although MCA theory enlightens one aspect of articulation-induced fluid recovery, it neglects at least one other important contributor to the phenomenon. In particular, when the contact area sits stationary relative to the cartilage, as in the case of tibial plateau of the knee and acetabulum of the hip, the resulting stationary contact area (SCA) provides no opportunity for osmotic swelling. Nonetheless, in the last few years, experimental studies have shown that cartilage recovers interstitial fluid within loaded SCAs during sliding without any benefit from contact migration or unloading: these experiments show that recovery can be purely sliding-induced rather than unloading-induced or migrationinduced, and this specific mode of recovery has been defined as "tribological rehydration". Tribological rehydration occurs, by definition, at the contact interface, which involves multiple roughness scales as shown in Figure 1 [18]. Experiments show that the tribological rehydration phenomenon crucially determines the most basic conditions of joint contacts, but no theoretical justification of the process or of its implications on the system tribology has been so far provided.

In this paper, we give the first answer to this question by developing an innovative strategy to deal with cartilage lubrication. In detail, we employ a Finite Element (FE) model to study, at the macro-scale, the loading-induced exudation and, then, by moving from the configuration provided by the FE solver, we focus on the porous flow dynamics within the contact interface. Our proposed approach accounts for the micro-roughness of cartilage and fluid flow within percolated interfacial pathways. We discuss the results within the context of experimental observations and conclude that sliding-induced rehydration has, indeed, an hydrodynamic origin. Providing an explanation to long-standing issues related to rehydration will, ultimately, furnish useful tools to optimize the function of native cartilage and to delay the onset or mitigate the risks of osteoarthritis. Furthermore, a deeper understanding of the rehydrative mechanisms will also guide the development of new biomaterials designed accounting for tribological rehydration as one of their key criteria: this is vital to succeed in the production of new disruptive technology and synthetic solutions for cartilage replacement [19][20].

\section{Materials and methods}

The methodology developed in this work and detailed in this Section aims at addressing the most important limitations that have so far hindered our ability to explicitly model and quantify the physical origin of the sliding-induced rehydration mechanism in articular cartilage. As schematically depicted in Figure 2, this requires us to capture not only the macroscopic behavior of the biphasic tissue as it is subjected to external loading and sliding, but also the intimately connected interaction between the fluid and the tissue as flow is established due to the pressure gradients established at the

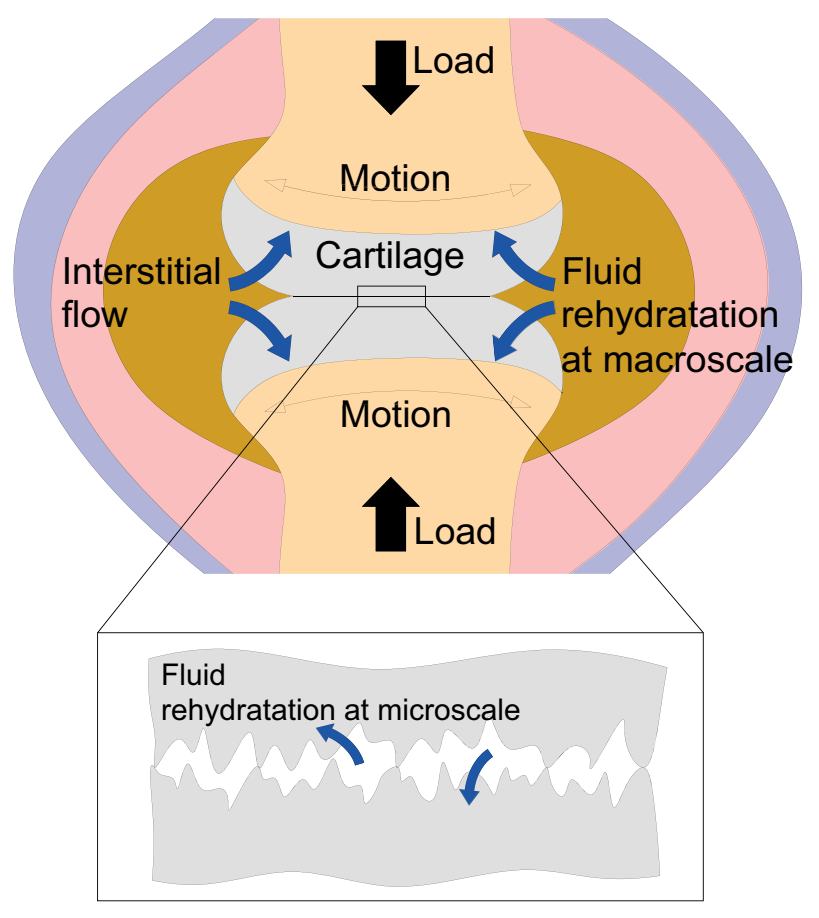

Figure 1: Schematic of the sliding-induced rehydration at the macro- and micro- scale. Due to relative sliding, cartilage tissues are rehydrated by the synovial fluid (dark yellow) contained, with the articulating bones, in the joint capsule. The latter includes the synovial membrane (pink), which secretes the synovial fluid, and the outer fibrous membrane (violet), which may contain ligaments.

contact edges. This is a key aspect to consider as it governs the rehydration of the tissue, whose behavior is then also dictated by the capability of the tissue to maintain "lubrication" throughout the contact interface. Furthermore, we argue that the interplay between surface deformation and fluid flow in the contact zone must be described adopting a multiscale approach that describes the effect that asperities and micro-contacts have on the overall flow so that the coupling between the fluid motion outside and inside the tissue is correctly captured everywhere within the contact. Accounting for roughness at the contact interface is crucial not only to guarantee the accuracy of the model, but, most fundamentally, as elucidated later in the paper, to guarantee lubrication throughout the entire joint. It should be noted, in fact, that continuity of flow must be maintained to observe rehydration when the contact problem suggests operating conditions commonly in the mixed lubrication regime, i.e. when the interface is characterized by a percolated network of interfacial gaps rather than by a full fluid film that separates the contacting surfaces. This means that, as suggested by previous theoretical studies, without considering the details of fluid transfer to and from the tissue and the pseudo-equilibrium established at the asperities level, it is not possible to correctly model and predict the overall tissue response [21][22][23].

The modelling strategy and its components are presented in the next sub-section before introducing the experimental 
evidence used to corroborate the results of our simulations.

\subsection{Finite Element simulation of cartilage dehydration}

In order to shed light on the physical mechanism that triggers cartilage sliding-induced rehydration, it is necessary to first assess what occurs when this porous tissue is statically loaded and, thus, undergoes dehydration [15][27][28]. Indeed, this is crucial as it will be used as a starting point for the study of rehydration. Here, the draining process due to static loading is modelled by using the FEM software package Abaqus v. 2017 [29][30][31]. Let us observe that, at this stage, we neglect the role played by the roughness: indeed, the micro-squeeze occurring due to the static loading [32][33] does not provide qualitatively significant variation to the loading problem solution in terms of stress/strain distribution, of contact pressures and, ultimately, of the entire biphasic mechanics. Consequently, as reported in Figure 2a, by reproducing a contact configuration mimicking the typical experimental setup [34], we model a smooth cartilage cylindrical punch in contact with a flat disk assumed to be perfectly rigid.

In detail, we adopt the built-in soil consolidation procedure available in Abaqus to deal with biphasic porous materials [30][31]. Modelling articular cartilage as a perfectly biphasic material has been pioneeringly proposed by Mow et al.[35][36] and is indeed an idealized approach [37][38] as, in reality, cartilage tissues are a saturated mixture including a fluid phase, a porous solid extra-cellular matrix and three-dimensional fibrils closely embedded in the solid [39]. The real cartilage rheology is likely to play a role in the system dynamics, including the diffusion, after rehydration is started, of the synovial fluid into the tissue, but, to understand the original triggering mechanism of rehydration, that is the scope of the paper, a simple biphasic constitutive description of cartilage is a fair and useful approximation. Indeed, including into the analysis additional variables for the solid behavior, such as tension-compression nonlinearity, viscoelasticity, anisotropy, and heterogeneity, and, eventually, other non-linear effects for the synovial fluid, like a non-Newtonian rheology, could, in fact, potentially mislead our analysis from distinguishing causes and effects, failing in isolating the real origin of the phenomenon under investigation. As a consequence, the numerical model employed in this study is a classic biphasic model [20], where the material constitutive behavior is fully governed by three parameters, consisting in the Young's modulus $E$, the Poisson ratio $v$ and the permeability $k$. Specifically, in the analysis carried out in this study,consistently with what widely available in literature (see e.g. Ref. [40]), we assume an elastic modulus $E$ equal to $E=0.55 \mathrm{MPa}$. With regards to the Poisson ratio $v$, as cartilage behaves like a collapsing network of fibres, for the solid component in the biphasic material, we assume $v$ equal to $v=0$. As for the permeability $k$, again coherently with specific studies in literature [40], $k$ is assumed equal to $k=4.2 \cdot 10^{-12} \mathrm{~mm}^{2}$. Finally, for the fluid, we employ a viscosity $\eta$ equal to $\eta=0.001 \mathrm{~Pa} \cdot \mathrm{s}$. We implement a plain strain $2 D$ configuration, with the cartilage circular segment, having a radius $R=1 \mathrm{~m}$ and the sagitta $s=280$ $\mathrm{mm}$, in contact with a rigid analytical surface. Let us notice that the results are later presented in dimensionless form and will, then, applicable to any particular joint geometry. Fluid is allowed to exude on the entire arc length, but we impose specific boundary conditions once the contact arises: specifically, as no micro-exudation is considered, the contact area is assumed sealed, whilst free draining can occur in the non-contact region [27][28]. The numerical analysis, which employs 8 node pore pressure elements (CPE8RP) for the cartilage tissues, is carried out in displacement control: specifically, a displacement $d$ being equal to $d=1 \mathrm{~mm}$ is applied to the top surface of the cartilage (Figure 2a). The contact is assumed as frictionless.

\subsection{Percolation-based approach to mixed lubrication in porous media}

The crucial purpose of this paper is to assess the physical mechanism that guarantees a sliding-induced rehydration in articular joints. Consequently, we focus on the system configuration reported in Figure $2 b$, where, after applying the normal load, the rigid substrate starts to slide, thus triggering the rehydration process.

With regards to the lubrication dynamics, this can be studied by means of the Reynolds equations [46][47], based on the mass conservation and properly modified to account for the source term related to the flow given to or taken from the porous solid. In particular, by neglecting the fluid compressibility, as usually done when dealing with soft contacts, we can write:

$$
\nabla \cdot \mathbf{J}_{l}=V_{p}
$$

where $\mathbf{J}_{l}$ is the lubricant volumetric flow and $V_{p}$ is the flow that is exchanged with the porous medium and is defined as porous flow. Classical considerations on flow conservation lead to determine the first term as [46]:

$$
\mathbf{J}_{l}=-\frac{h^{3}}{12 \eta} \nabla p+\frac{\mathbf{u}}{2} h
$$

where $h$ is the lubricant film thickness, $\eta$ is the viscosity of the lubricating fluid, $p$ and $\mathbf{u}$ are respectively the fluid pressure and the entrainment speed.

By assuming that inertial effects are negligible, the porous flow can be, on the other hand, obtained by means of the Darcy law [48], regulating the fluid dynamics in the porous flow:

$$
V_{p}=-\frac{k}{\eta} \nabla p_{p} \cdot \mathbf{n}
$$

with $\mathbf{n}$ being the unit vector being normal to the porous surface, and $p_{p}$ being the pore pressure in the biphasic body. For continuity reasons, at the boundary of the porous solid, $p$ and $p_{p}$ have to be equal. 


\section{Schematic model}
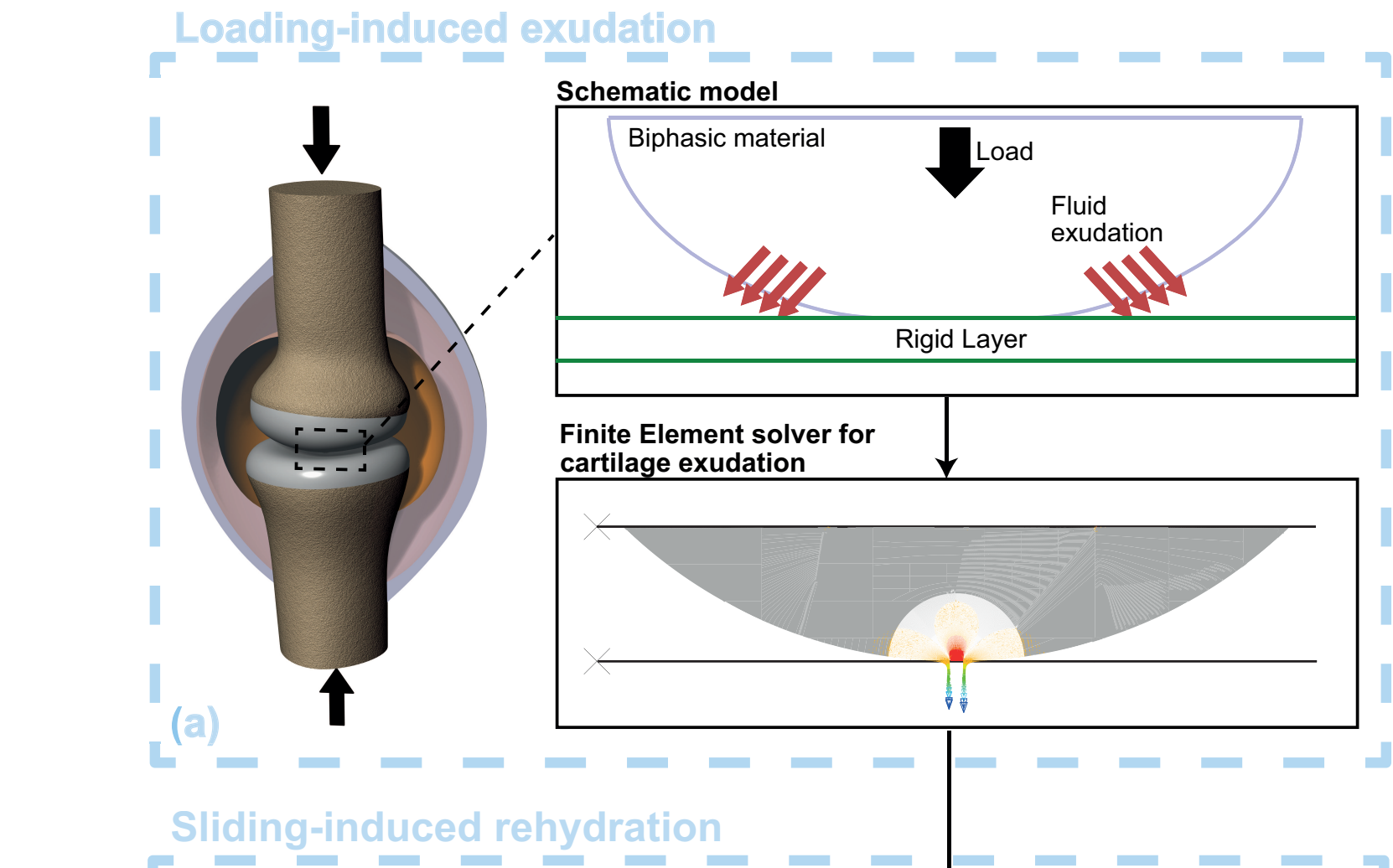

\section{Schematic model}

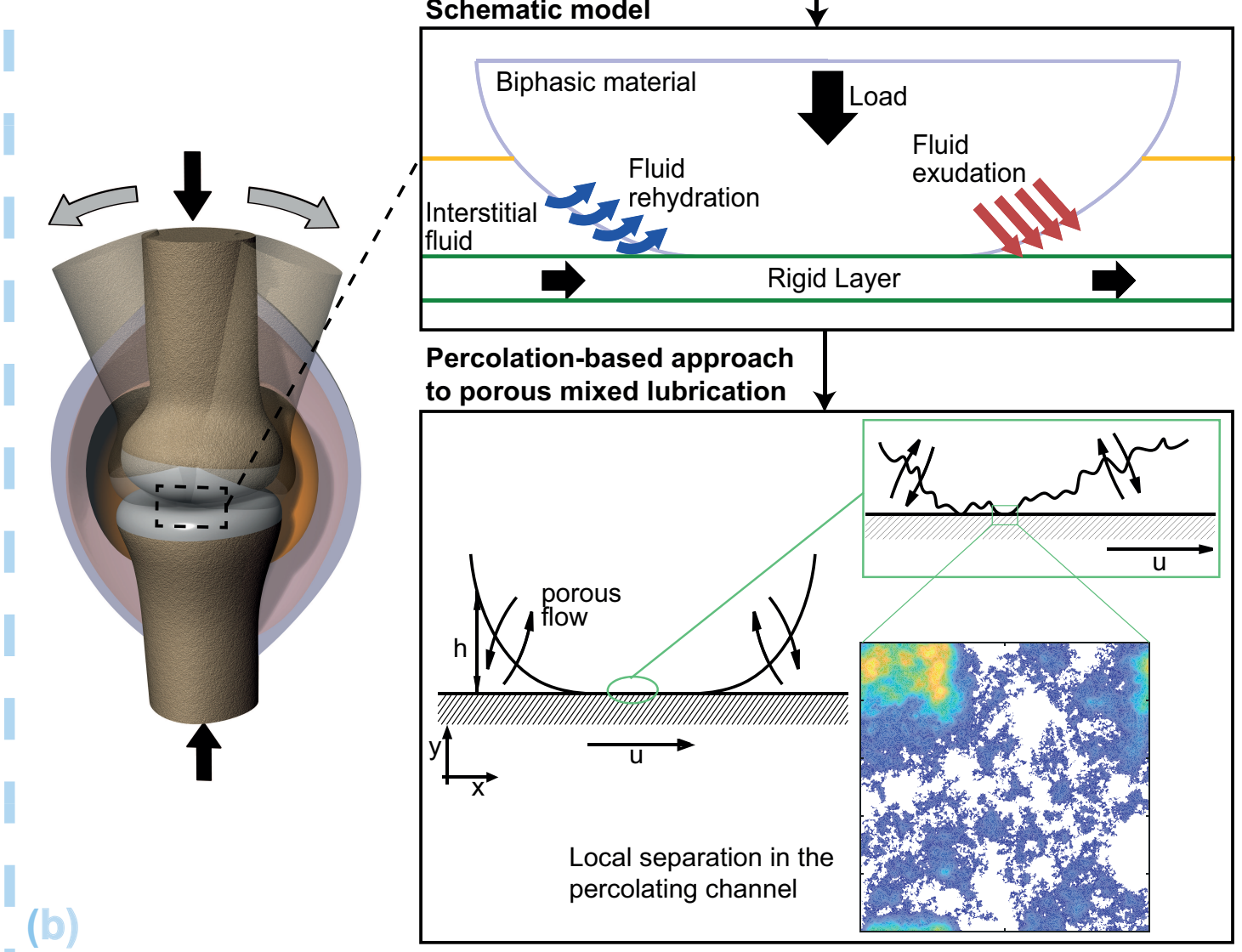

Figure 2: Conceptual schematic of the approach employed in this study. Loading-induced exudation is assessed by means of a Finite-Element model (a), while the sliding-induced rehydration, sketched on the bottom (b), is studied by developing a percolation-based numerical approach to deal with the complex mixed lubrication regime occurring at the interface. 
Let us, now, observe that, as we are focusing on a twodimensional configuration, Eq. (1) can be further simplified [49]. Specifically, we can express the volumetric flow $\mathbf{J}_{l}$ as $\mathbf{J}_{l}=J_{l} \mathbf{i}$, with $\mathbf{i}$ being the unit vector corresponding to the $x$-axis and $J_{l}$ being equal to $J_{l}=-\left(h^{3} / 12 \eta\right)(d p / d x)+$ $u h / 2$, where $u$ is the entrainment speed along the $x$-axis direction and, thus, $\mathbf{u}=u \mathbf{i}$. Furthermore, as we are going to focus on a two dimensional region, which, under deformation, can be approximated to flat, we consider that $\mathbf{n}$ coincides with the unit vector $\mathbf{j}$ referred to the $y$-axis. Thus, we can write $V_{p}$ as $V_{p}=-(k / \eta)\left(d p_{p} / d y\right)$.

After such manipulations, Eq. (1) becomes:

$$
\frac{d J_{l}}{d x}=V_{p}
$$

Now, it should be noticed that, as schematically reported in Figure 2b, the system is in a severely mixed lubrication regime, i.e. it operates in a regime very close to boundary lubrication [46]. This means that, in the region macroscopically into contact, the average film thickness will be much smaller than the surface roughness [46]. Unfortunately, under these conditions, given the vast number of rough scales involved in the problem, a deterministic solution of Eq. (4) is extremely difficult to obtain [46]. As a consequence, a variety of statistical approaches has been developed to deal with mixed lubrication [50][51][52][53][54]: the common idea, shared by the majority of these approaches, is based on the introduction of so called flow-factors coefficients, which, once introduced in the modified Reynolds equation, accounts for the role played by the roughness.

As for the surface roughness, let us briefly recall that, from a statistical point of view, once the topography has been acquired, it is possible to introduce the power spectral density (PSD) $C(\mathbf{q})=(2 \pi)^{-2} \int d^{2} x\left\langle h_{c}(\mathbf{0}) h_{c}(\mathbf{x})\right\rangle \exp (-i \mathbf{q} \cdot \mathbf{x})$, where $h_{c}(\mathbf{x})$ is the height distribution and the symbol $\langle\cdot\rangle$ stands for the ensemble average [41][42][43]. Indeed, it is well known that all the statistically relevant information, including inter alia the possible surface anisotropy, are summed up in the spectral components of $C(\mathbf{q})$. Furthermore, determining the PSD of the cartilage samples is crucial also for generating statistically equivalent numerical surfaces. As we are going to see, these numerical realizations, whose generation technique can be found in Ref. [43], are necessary to obtain the micromechanical statistically relevant results needed to assess the governing lubrication mechanism. These numerical replica have the PSD of the original surface, but can be generated with a different number of scales $N$ and have, thus, spectral components in the range of wave vectors $q_{0}<|\mathbf{q}|<N q_{0}$ with $q_{0}=2 \pi / L_{0}$ being the roll-off vector related to the fundamental dimension $L_{0}$ of the rough sample. This allows us to fully replicate the multiscale lubrication involving roughness in a spectral range spanning several orders of magnitude.

Now, let us come back to the necessity of determining the flow factors to be inputted in Eq. (4) as this is necessary to assess the role that roughness plays in the lubrication dynamics. Here, we propose an original phenomenological approach based on percolation [55][56][57]. Indeed, let us start by observing what would happen if no roughness were accounted for and the contacting surfaces were considered perfectly flat: after concluding the loading process, no fluid would be present in the contact region and, even when the relative sliding is started, as this region would be practically sealed, no lubricating film could originate, and, thus, no hydrodynamic pressure, no lift-off force and, ultimately, no rehydration would occur. Fortunately, as the contacting surfaces are rough, some fluid is always entrapped at the interface and, in presence of sliding speed, there is lubrication. Precisely, we have a mixed lubrication regime, where the film thickness is smaller than the surface roughness. This is an intrinsically multiscale regime [22]. Now, to this extent, at a given magnification $\varsigma=N q_{0}$, the contact area will split into different contact clusters [23]: specifically, as shown in Figure 3a, below a given threshold, defined percolation threshold, it is possible to distinguish a percolating channel that goes through the entire reference domain [55]. Unlike the contact areas and the isolated non-contact clusters that do not percolate, the percolating channel enables the lubricant to flow, thus enabling flow continuity and lubrication. Indeed, from such a point of view, related to lubrication, as shown in Figure 3b, the crucial distribution to focus on is the local height in this channel, that is, the separation $s$ between the contacting surfaces in the percolating channel: ensemble average separation $\langle s\rangle$ and root-mean-square $s_{r m s}$ are, in particular, the variables that govern the phenomenon. Incidentally, let us notice that, as shown in detail in the Supplementary Information (Section S1), these quantities are obtained by solving the elastic problem at the micro-scale, by means of the Boundary Element Method described in Ref. [43]. Now, looking at the percolation process, when the load is increased and the percolation threshold is passed, contact areas grows so much that no percolation may occur. However, if the magnification $\varsigma$, at which the surface is observed, is increased, i.e., additional scales of roughness are included, the contact clusters fragment and a new percolating channel origin: as a consequence, it is possible to conclude percolation persists under all conditions given that the model includes an adequate number of scales. At the same time, as percolation come from the new scales which have been added, one should observe that the mean separation in the percolating channel $\langle s\rangle$ will be related to these smallest scales: as shown in detail in the Supplementary Information (Section S1) to this paper, $\langle s\rangle$ monotonically decreases as $\varsigma$ is increased. This is fully consistent with the physics of the problem as, by increasing the load and, thus, the real contact area, more scales have to be added to achieve percolation: $\langle s\rangle$ will decrease and the lubricant flow rate in the contact will reduce.

Now, this phenomenological relation between percolation and lubrication at the micro-scale plays a fundamental role in the homogenization of Eq. (4) and allows one to obtain a statistical assessment of mixed lubrication conditions. Indeed, let us define a corrected film thickness $h_{p}$ : the latter is equal, in the non-contact region, to the gap between 

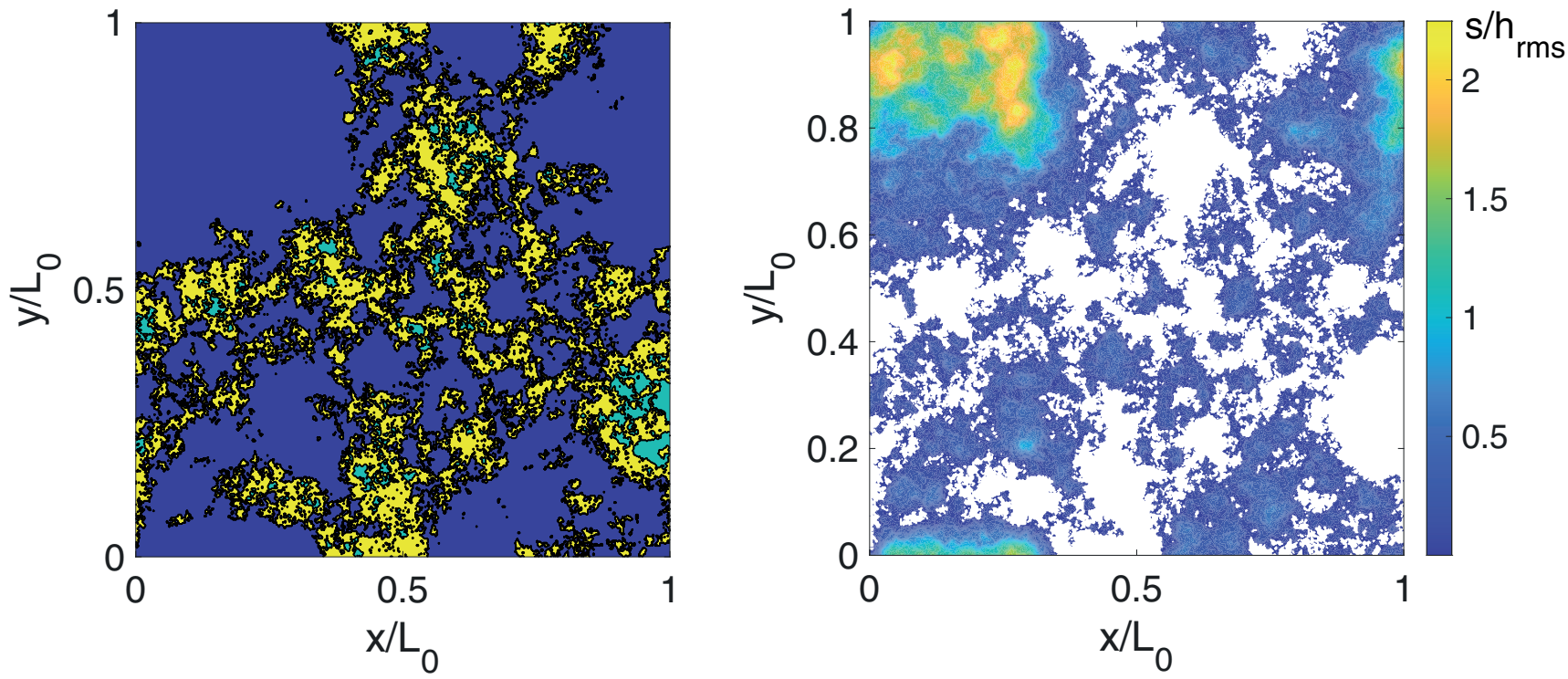

Figure 3: Solution of the micro-mechanical problem at the percolation threshold: (a) cyan regions refer to non-percolating non-contact region, adjacent yellow areas to contact clusters and, finally, we have the percolating channel in dark blue, and (b) local gap height $s(x, y) / h_{r m s}$ in the percolating channel.

the cartilage circular sector and the rigid layer (see Fig. 2), while, in the contact region, $h_{p}$ can be defined as the the mean separation $\langle s\rangle$ in the percolation channel at the magnification $\varsigma$, at which percolation is detected. Coherently, it is possible to define the root means square $h_{\text {prms }}$ being equal to the cartilage roughness rms in the non-contact macro-region and to $s_{r m s}$ in the contact area.

We can, then, define the average flow $\boldsymbol{J}$ as:

$$
J=-\varphi_{p} \frac{h_{p}^{3}}{12 \eta} \frac{d p}{d x}+\varphi_{s} \frac{u}{2} h_{p}
$$

where $\varphi_{p}$ and $\varphi_{s}$ are respectively the pressure and the shear flow factors. In the case of isotropic rough surfaces, $\varphi_{p}$ and $\varphi_{s}$ are almost equal [50][51]: given the mainly qualitative purpose of this study, we will assume $\varphi_{p}=\varphi_{s}=\varphi_{\text {fluid }}$ . Furthermore, in order to quantify $\varphi_{\text {fluid }}$, let us define $\lambda$ as the ratio $\lambda=h_{p} / h_{\text {prms }}$ : moving from simple dimensional considerations (the reader is referred to [54]), it is possible to assume that, for $\lambda>>1, \varphi_{\text {fluid }} \approx 1-1 / \lambda^{2}$, whereas for $\lambda$ going to $0, \varphi_{\text {fluid }}$ must vanish. Between these two limits, it is easy to extrapolate a qualitative trend for $\varphi_{\text {fluid }}$ as a function of $\lambda$.

The presence of the roughness will, obviously, impact also the porous flow $V_{p}$. In particular, we assume that the biphasic solid can exchange fluid just in the percolating channel and, thus, we write the average porous flow $V$ as:

$$
V=-\varphi_{p o r} \frac{k}{\eta} \frac{d p_{p}}{d y}
$$

with $\varphi_{\text {por }}$ being the porous flow factor defined as the ratio $\varphi_{\text {por }}=A_{C} / A_{0}$ between the the percolating channel area, $A_{C}$, and the nominal contact area, $A_{0}$.
Ultimately, without any loss of generality, but observing that in soft contacts we usually consider the fluid as isoviscous, we can rewrite Eq.(4) as:

$$
\frac{d}{d x}\left(\varphi_{\text {fluid }} h_{p}^{3} \frac{d p}{d x}\right)=6 \eta\left[\varphi_{\text {fluid }} u \frac{d h_{p}}{d x}+\varphi_{\text {por }} \frac{k}{\eta} \frac{d p_{p}}{d y}\right]
$$

Eq.(4) is the homogenized equation determining the lubrication of the system in Figure $2 b$ and should be coupled with the relations governing the biphasic solid problem. However, since in this paper we are interested in enlightening the cause that sets off the rehydration process, rather than assessing the entire lubrication process, for simplicity reasons, we consider the fluid and the solid mechanics as uncoupled. We will, then, solve Eq. (4) in a configuration that is solely and exhaustively determined by the solid solver at the end of the loading stage. This means that, out of the nominal global contact area, $h_{p}$ is given by the Abaqus FE deformation, whereas, in the contact region, $h_{p}$ is determined, as average separation in the percolating channel, by a Boundary Element solver following the approach explained in detail in the Supplementary Information SI, Section S1.

Notwithstanding the approximation intrinsically implied in uncoupling the solid constitutive mechanical problem and the fluid lubrication, this approach will enable a clear view of what happens, in terms of fluid given by or provided to the biphasic solids, when relative sliding between contacting bodies is set.

\subsection{Experimental testing}

Experimental measurements are conducted to corroborate the aforementioned theoretical framework. The experimental setup, mirroring those successfully employed in previous studies [34], is sketched in Figure 4. Briefly, three 


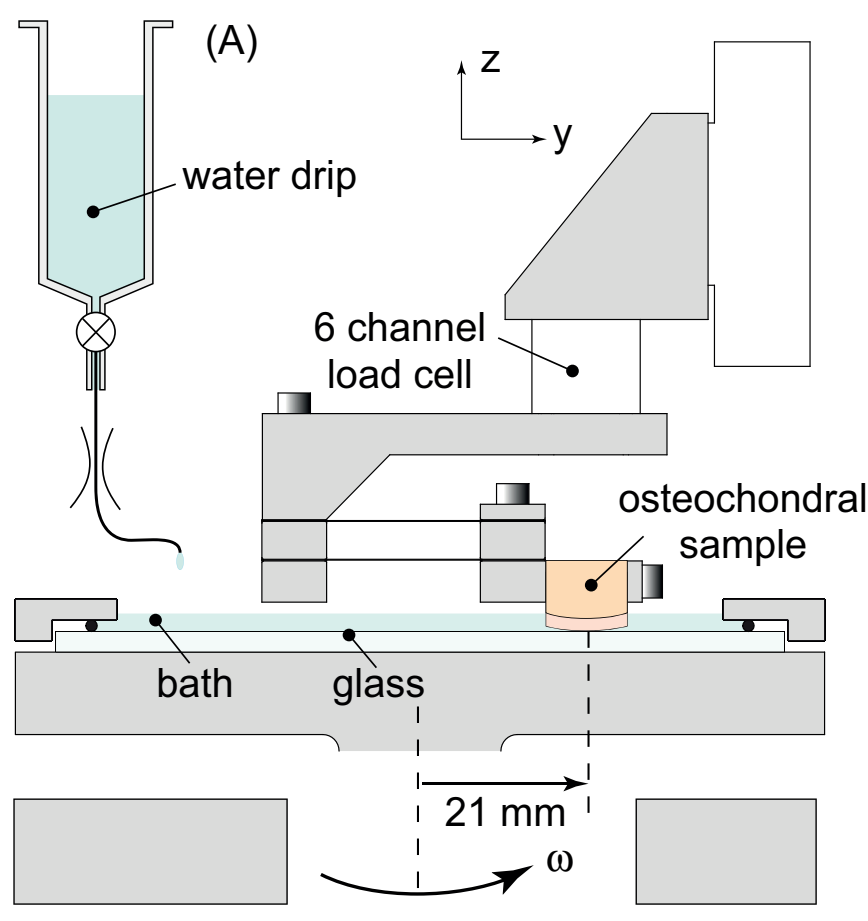

Figure 4: Schematic of the experimental setup developed to assess cartilage rehydration.

bovine stifles are obtained from a local abattoir and dissected. We have then used a $19 \mathrm{~mm}$ diameter coring saw to extract $N=6$ explant samples, which have been rinsed, stored in, and lubricated by a solution of protease inhibitor (P2714, Sigma Aldrich) dissolved in phosphate buffered saline to a dilution of $1 \mathrm{X}$ based on the manufacture's recommendations (PBS-PI). Samples have been stored at $4 \mathrm{C}$ and tested within 4 days of harvest. To set up the experiment, samples have been loaded into a custom pin-on-disc tribometer with a PBSPI bath (see Figure 4). The sample center has been located at a radius of $21 \mathrm{~mm}$ from the center of disc rotation and the reported speed is the product of this radius and the measured rotational speed. Loads have been applied with a precision nanopositioning stage and measured with a 6-channel load cell; a linear encoder $(\hat{\mathrm{A}} \pm 100 \mathrm{~nm})$ on the stage has been used to track the compression and recovery response of cartilage over time.

As with the theoretical approach, fluid has been first driven from the sample with a period of static loading; here we used a load of $5 \mathrm{~N}$ for 90 minutes. Following exudation, disc rotation was used to induce tribological rehydration while maintaining the constant $5 \mathrm{~N}$ load. The tribological rehydration rate was quantified using the rate of fluid recovery over the first 10 seconds of sliding. Following 20 minutes of sliding, the sample was unloaded and allowed to free swell for 10 minutes. The process was repeated for other randomly selected speeds from the following group: $1,5,10,15,20,30$, 50,75 , and $100 \mathrm{~mm} / \mathrm{s}$. Not all samples slid at every speed and some speeds were repeated on a single sample for repeatability.
Finally, in order to characterize the topography of each sample, and, thus, to obtain all the information useful to implement our lubrication approach, white light interferometer consisting in a Wyko NT9100, Veeco with a 20x magnification objective is employed. Let us notice that, in order to use WLI, the surface has to be completely dry in order to avoid light reflecting from the fluid film rather than from the cartilage surface. Thus, the surface of the samples has been gently patted dry using a soft absorbing paper to remove surface fluid without compromising subsurface hydration or the asperity scale topography of interest. In order to obtain statistically significant measures, we take five different measurements in adjacent areas of the tissue: the imaged area, for each scan, is of $230 \times 230 \mu \mathrm{m}^{2}$.

\subsection{Statistical analysis}

All the statistical analyses on the experimental data are performed by means of the software Matlab version 2020a (Mathworks). In particular, the results are shown by means of whisker boxes: on each box, the central mark refers to the median, and the bottom and top edges of the box indicate respectively the 25 th and 75 th percentiles [58]. It should be noted that the whiskers extend to the most extreme data points that are not considered outliers: the outliers are plotted individually using the '+'symbol.

\section{Results}

The analysis of the cartilage samples, whose details can be found in the Supplementary Information (Section S2), confirms that cartilage surface roughness conforms to the assumption embedded in the homogenization approach developed in this paper. In particular, the probability density function of the height distribution is very well described by a Gaussian trend, while the power spectral density $C(\mathbf{q})$ is, in a log-log plot, linear, thus confirming that the surface can be successfully described by a self-affine model [43]. Furthermore, an analysis of the anisotropy characteristics reveals that the surface can be considered statistically isotropic. All this means that we can safely employ numerically generated isotropic self-affine surfaces to compute the homogenized quantities to input into Equation Eq.(4).

Indeed, by using the information obtained at a micromechanical level, we have calculated the flow factors $\varphi_{\text {fluid }}$ and $\varphi_{\text {por }}$ in the entire domain; thus, we have been able to focus on the solution of Eq. (4). In Figure 5, we show the results in terms of dimensionless pressure $p R / \eta u$, dimensionless volumetric flow $J R / u$ and dimensionless porous flow $V / u$. In detail, we observe that the pressure distribution has a welldefined peak at the contact inlet, but later it drops assuming the more standard parabolic distribution in the contact area. Such a trend is clearly due to a wedge effect: indeed, pressure has to sharply increase following the reduction of the film thickness; in the contact area, however, where the film thickness reduces and depends only on the roughness, the pressure has to decrease and, by conforming to the pore and contact pressure distributions, acquires an Hertzian-like shape. Indeed, at the contact outlet, where the film thickness 


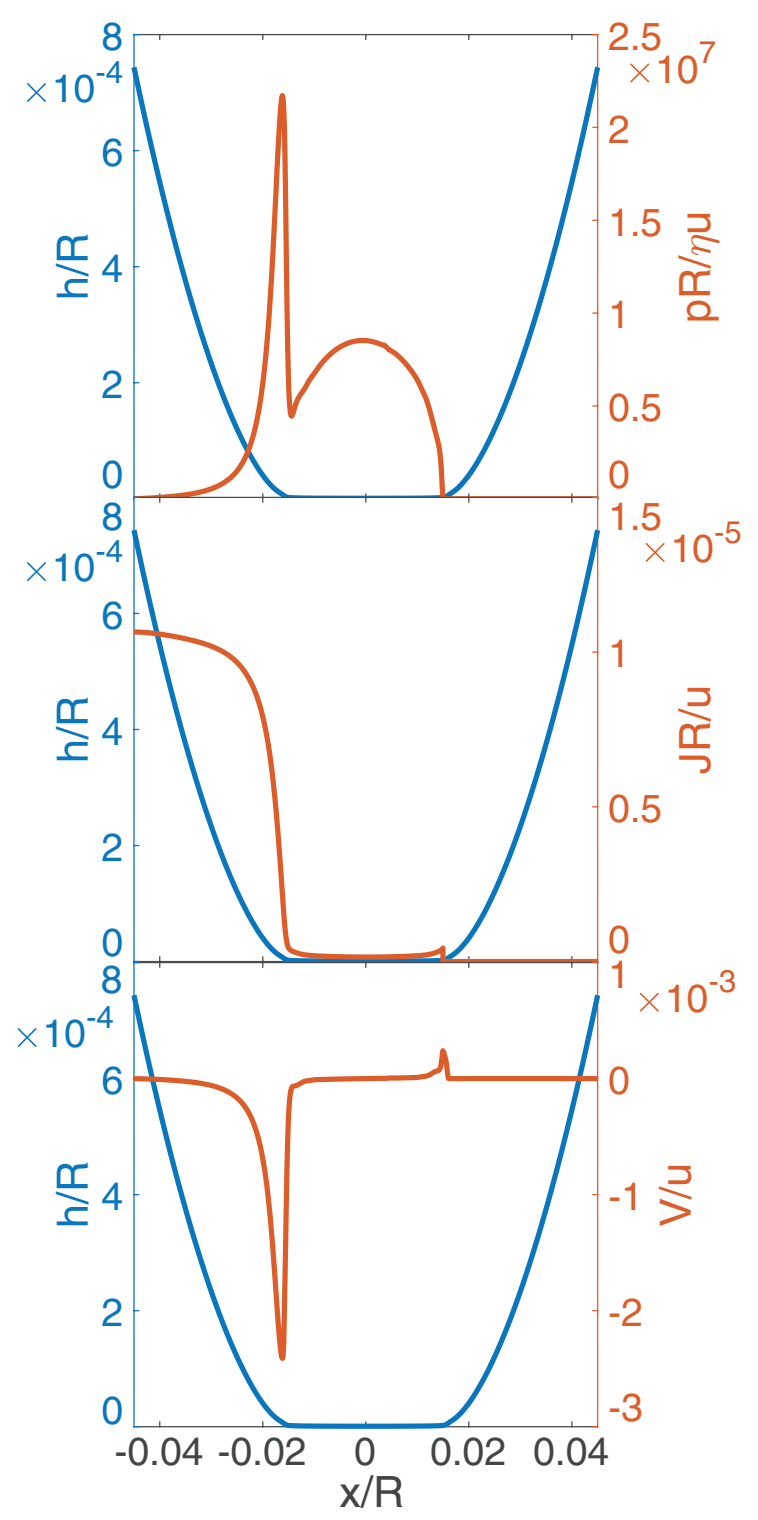

Figure 5: Dimensionless pressure $p R / \eta u$, dimensionless volumetric flow $J R / u$ and dimensionless porous flow $V / u$ along the lubricated region $x / R$. On left $y$-axis, we report the deformed profile $h / R$.

increases again and the fluid exudes out, the pressure has to vanish.

All this has, obviously, implications on both the volumetric and the porous flows. Indeed, because of the sharp pressure peak at the inlet, we have a net flow $V / u$ that goes into the biphasic solid and, accordingly, the volumetric flow $J R / u$ has a marked decrease. The physical consistency is clear: due to the wedge effect and the relative pressure growth, part of fluid is conveyed into the biphasic solid and, thus, the flow rate of the lubricating fluid has to drop. In the contact area, where the roughness crucially determines the mixed lubrication regime, the porous flow $J R / u$ decreases to a small value, but it is not null as it keeps on lubricating the contact region. Interestingly, in this zone, $J R / u$ has a very slight

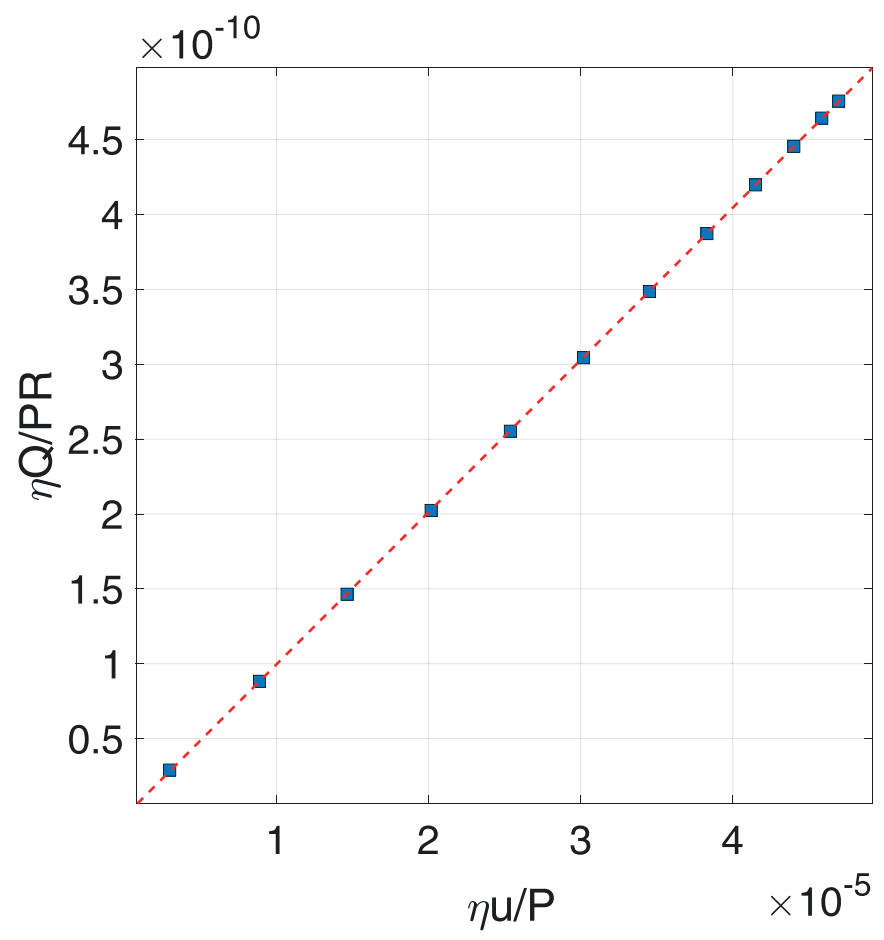

Figure 6: Dimensionless global flow $\eta Q / P R$ as a function of the sliding dimensionless speed $\eta u / P$. Blue squares refer to numerical outcomes, while red dotted lines to linear fit.

variation and, thus, $V / u$ is extremely small. Differently, at the outlet, where the flow pressure goes to zero due to the fluid expansion, the biphasic solid keeps on experiencing a certain level of exudation: $J R / u$ increases, while a porous flow $V / u$ comes out from the solid.

The crucial aspect here is that, because of the relative sliding occuring between the contacting bodies and the relative lubrication mechanism, there occurs a marked rehydration in the biphasic solid as a direct consequence of the peak the porous flow $V / u$ occuring at the fluid inlet. Such a rehydration effect is, indeed, global and, as we will see, is speeddependent. To this end, let us define the global flow $Q$ exchanged with the solid as the integral of $V$ over the entire contact length:

$$
Q=-\int_{a} V d x
$$

It should be noted here that, in order to simplify the interpretation of the results, we have defined the global flow $Q$ in such a way that, unlike $V$, it will be positive when a net flow goes into the solid. In Figure 6 , where we plot the dimensionless quantity $\eta Q / P R$ as a function of the sliding dimensionless speed $\eta u / P$, with $P$ being the total normal load, the global flow grows linearly with the sliding speed, thus revealing that rehydration, in the cartilage configuration in Fig. 2b, has a hydrodynamic origin. This theoretical prediction is qualitatively similar to the experimental trends shown in Figure 7. Indeed, on the experimental side, in Fig- 
ure $7 \mathrm{a}$, we measure, at sliding speeds ranging from $1 \mathrm{~mm} / \mathrm{s}$ to $100 \mathrm{~mm} / \mathrm{s}$, the compression as a function the time: due to the rehydration, compression tends to decrease. The slope of each curve, after $10 \mathrm{~s}$ of motion, is defined as the rehydration rate $S$ at the onset of sliding, that is, the speed at which the bone moves away from the substrate when relative sliding is triggered. In Figure 7b, we plot $S$ as a function of the sliding speed $u$ and we find a linear trend fully consistent with theoretical outcomes. The coefficient of determination $R^{2}$ is, in this case, equal to $R^{2}=0.8$.

\section{Discussion}

As it is demonstrated extensively by existing literature and supported by our FE analysis, static load-carrying articular joints experience significant synovial fluid exudation and, thus, marked joint space thinning. Fortunately, even loaded joint movement following static loading is known to reverse the exudation process through a combination of osmotic swelling at free surfaces and tribological rehydration within the loaded contact area: this ability of joints to recover interstitial fluid and thicken in response to movement is well-known, crucial to the long-term prevention of dysfunction, and of unclear origins. The long-standing scientific consensus is that joint movement periodically exposes the dehydrated surface to the bath, which automatically activates osmotic recovery at free surfaces [25][26]. Free swelling of this nature is widely regarded as the fastest possible rates of recovery [6][61] and, therefore, provides a sensible explanation of the observable recovery phenomenon. Until recently, sliding in the SCA was predicted and widely believed to inevitably defeat interstitial pressure and cartilage function regardless of conditions [21]. However, several years of experimental work demonstrating marked fluid recovery within the cSCA refutes that hypothesis and demonstrates that biphasic theory alone is inadequate for the analysis of these inherently tribological joints [34]. Our theoretical analysis here is the first to show meaningful sliding-induced fluid recovery within the loaded contact area.

Our results show, in particular, that tribological rehydration likely has a hydrodynamic origin and is closely related to the wedge geometry at the fluid inlet. These theoretical findings are consistent with the hypothesis posed by Burris and Moore to explain their experimental observations. Specifically, they proposed that hydrodynamic pressures build within the convergent wedge, peak near at the wedge tip, and propagate a rehydration front across the contact interface. Furthermore, let us observe that the predicted triggering rates are consistent with those observed experimentally. Indeed, according to our theoretical predictions in Figure 6, the ratio $Q / R u$ is approximately equal to $10^{-5}$. Now, the experimentally measured rehydration rate $S$ can be easily related to the global flow $Q$ as $Q=S a$ with $a$ the contact diameter: as in the experimental setup the condyle radius is $20 \mathrm{~mm}$ and the contact diameter is approximately 10 $\mathrm{mm}$, the rate $S a / R u=Q / R u$ varies experimentally between between $0.25 \cdot 10^{-5}$ and $0.9 \cdot 10^{-5}$. Although a one-
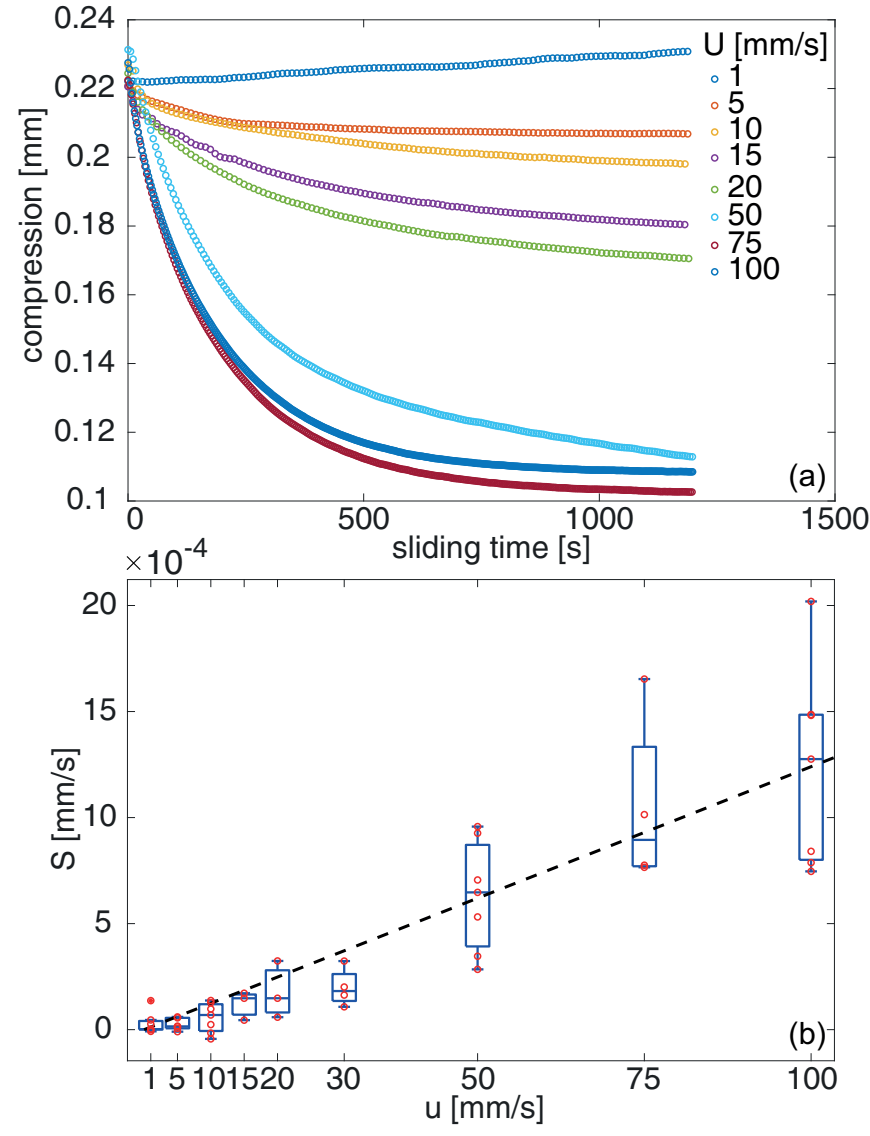

Figure 7: Compression as a function the time at different sliding speed values $u$ on the top (a), and the triggering rehydration rate $S$ as a function of the sliding speed $u$ on the bottom (b). The rehydration rate is obtained as the slope of the compression curves after $10 \mathrm{~s}$. On each box, the central mark refers to the median, and the bottom and top edges of the box indicate respectively the $25 \mathrm{th}$ and $75 \mathrm{th}$ percentiles [58]; red points refer to each experimental test.

to-one comparison between theory and experiments is out of the scope of the this paper, which is fully focused on enlightening the physical mechanism behind rehydration, this consistency in the rehydration rates - with $10^{-5}$ as theoretically estimated rate versus an experimentally measured value in the range $\left[0.25 \cdot 10^{-5}, 0.9 \cdot 10^{-5}\right]$ - is remarkable, especially given our inability, as a community, to anticipate tribological rehydration before its experimental discovery.

Furthermore, a recent experimental study by Graham et al. has tracked fluorescent solutes to more directly map the spatial characteristics of the tribological rehydration process [62]. Those experiments have shown that the recovered solutes are highly concentrated at the leading edge initially and distribute across the contact interface over time as shown in the inset of Figure 8. Our model is developed under steadystate conditions, but, again on qualitative basis, we can focus on a cycle, where the substrate moves sinusoidally over a pe$\operatorname{riod} T$, and, thus, we can define, in each point of the contact 


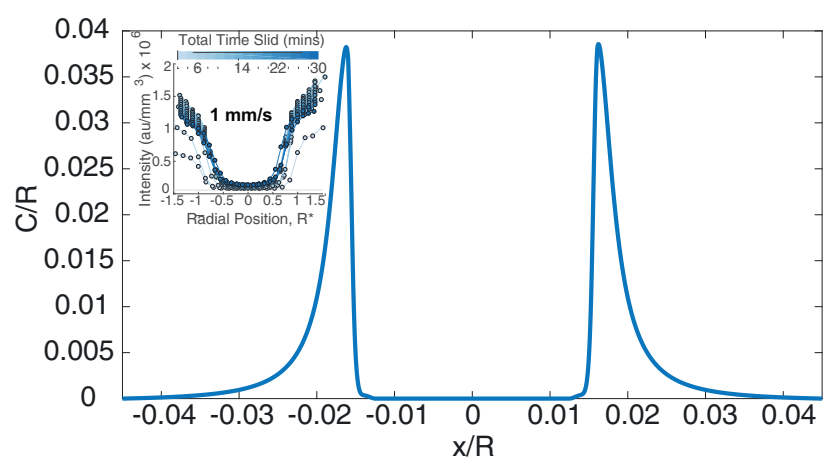

Figure 8: Cumulated flow $C / R$ along the contact region $x / R$. In the inset, reproduced with permission from Ref. [62], we report the experimental data referred a fluorescence test: point refers to the doping particles accumulation in the tissue.

region, the cumulative flow $C$ as:

$$
C=-\int_{T} V d t
$$

In spite of the approximation intrinsically embedded in employing a steady-state model for a reciprocating contact problem, Figure 8 reveals a strong coherence between experiments and numerical outcomes as the cumulated flow presents two peaks at the edges of the contact area. As seen before, at the edges, due to a wedge effect and the relative pressure peaks, the lubricant is conveyed into the solid: although we do not study the fluid diffusion from the edges towards the center of the contact region, and, indeed, we do not account for all the possible solid non-linearities, including for example viscoelasticity [63][64], the model provides an ultimate assessment of the rehydration triggering mechanism. We may conclude that, in natural articular joints and convergent stationary contact areas, tribological rehydration occurs, is activated by hydrodynamic pressure originated from sliding, and starts at the leading edge of contact.

\section{Conclusions}

In this work, we have develop an original strategy to deal with mixed lubrication between porous bodies. In particular, after studying the exudation process due to normal loading, we have defined a statistically homogenized relation where we relate the variation of the average lubricating flow with the porous flow, i.e., with the flow exchanged with the biphasic solid. Such an homogenized strategy is based on the definition, at multiple scales of roughness, of percolated interfacial gaps, where the fluid can flow ensuring lubrication. Thus, the homogenized Reynolds equation is crucially dependent on the mean separation and its rms in these non-contact percolating clusters. These quantities have been obtained by solving, at the micro-scale, the elastic rough problem [43]. On this basis, we have introduced proper flow factors for both the lubricanting and the porous flows.
Such an approach, originally proposed here to deal with severely mixed lubrication conditions, has allowed us to studyat least, qualitatively- the lubrication between a porous cylinder, mimicking the cartilage tissue, and a rigid substrate. This configuration reproduces the so-called convergent stationary contact area (cSCA), where experiments have shown that rehydration is sliding-induced rather than unloading or migration induced. Our analysis here shows that the slidinginduced entrainment of lubricating fluid into the converging wedge leads to hydrodynamic pressurization, and pressureinduced flow into the porous solid, particularly at the tip of the wedge. Let us underline that all this is possible thanks to the presence of a rough interface, which allows the lubricant to flow, at multiple scales, across the entire domain, including the area appearing, macroscopically, in contact.

Indeed, our study shows, on a theoretical basis, why articular motion reverses cartilage exudation and prevents tissue dysfunction. The theoretical solution predicts the most important and unpredictable experimental observations made to date: (1) cartilage recovers lost interstitial fluid within the loaded contact area during sliding and without contributions from unloading or migration; (2) fluid recovery rates are maximal near the tip of the convergent wedge; (3) the initial tribological rehydration rate increases proportionally with sliding speed. In addition to these qualitative trends, we predict approximately the same rates of tribological rehydration that are observed experimentally.

In addition to the theoretical interest in enlightening this mechanism, this numerical framework provides a practical starting point for the analysis of healthy joint mechanics under realistic tribological conditions. With further development, it will also provide much needed theoretical insights about the level of activity an average person should maintain to prevent excessive fluid loss, mechanical dysfunction, and eventual tissue failure. Furthermore, a better understanding of biphasic material lubrication will provide a significant contribution to engineer new self-lubricating biphasic materials, which would have a paramount impact in joint replacement and, in the industrial field, in slow-moving boundary lubricated bearings encountered in many engineering applications.

\section{A. Supplementary information}

Supplementary Information to this paper includes further details regarding the percolation-based homogenization approach here employed (Section S1) and the micromechanical characterization of the cartilage surface roughness (Section S2).

\section{Acknowledgement}

The authors acknowledge the partial support received from the Italian Ministry of Education, University and Research (Program Department of Excellence: Legge 232/2016 Grant No. CUP-D94I18000260001) [C.P.], the NSF BMMB program (grant number 1635536 and 1937493) [D.B. and 
A.M.], the Engineering and Physical Science Research Council (EPSRC) through the Established Career Fellowship EP/ N025954/1 [D.D.].

\section{References}

[1] Baker M.I., Walsh S.P., Schwartz Z., Boyan B.D., A review of polyvinyl alcohol and its uses in cartilage and orthopedic applications. Journal of Biomedical Materials Research Part B: 2012:100B,14511457, (2012).

[2] Pitsillides A. and Beier F., Cartilage biology in osteoarthritis-lessons from developmental biology. Nature Reviews Rheumatology, 7, 654, (2011).

[3] Makris E., Gomoll A.H, Malizos K.N., Hu J.C., Athanasiou K.A., Repair and tissue engineering techniques for articular cartilage. Nature Reviews Rheumatology, 11, 21, (2014).

[4] Murphy L.B., Cisternas M.G., Pasta D.J., Helmick C.G., Yelin E.H., Medical expenditures and earnings losses among US adults with arthritis in 2013. Arthritis Care \& Research (Hoboken), (2017).

[5] Cisternas M.G., Murphy L., Sacks J.J., Solomon D.H., Pasta D.J., Helmick C.G., Alternative Methods for Defining Osteoarthritis and the Impact on Estimating Prevalence in a US Population-Based Survey. Arthritis Care \& Research (Hoboken). 68(5):574-580, (2016).

[6] McCutchen C.W., The frictional properties of animal joints. Wear, 5:1e17, (1962).

[7] Armstrong C.G., Lai W.M., Mow V.C., An analysis of the unconfined compression of articular-cartilage. Journal of Biomechanical Engineering, 106(2):165e73, (1984).

[8] Oloyede A., Broom N., The generalized consolidation of articular cartilage: an investigation of its near-physiological response to static load. Connective Tissue Research, 31(1): 75e86, (1994).

[9] Jay G.D., Waller K.A., The biology of lubricin: near frictionless joint motion. Matrix Biology. 39: 17-24, (2014).

[10] Asanbaeva A., Masuda K., Thonar E.J., Klisch S.M., Sah R.L., Cartilage growth and remodeling: Modulation of balance between proteoglycan and collagen network in vitro with $3 \mathrm{~b} 2$-aminopropionitrile. Osteoarthritis and Cartilage. 16 (1): 1-11, (2008).

[11] Fife R.S., Brandt K.D., Braunstein E.M., Katz B.P., Shelburne K.D., Kalasinski L.A., et al., Relationship between arthroscopic evidence of cartilage damage and radiographic evidence of joint space narrowing in early osteoarthritis of the knee. Arthritis \& Rheumatology, 34(4):377e82, (1991).

[12] Herberhold C., Faber S., Stammberger T., Steinlechner M., Putz R., Englmeier K.H., Reiser M., Eckstein F., In situ measurement of articular cartilage deformation in intact femoropatellar joints under static loading, Journal of Biomechanics 32 , 1287-1295, (1999).

[13] Cooper C., Snow S., McAlindon T.E., Kellingray S., Stuart B., Coggon D., et al., Risk factors for the incidence and progression of radiographic knee osteoarthritis. Arthritis \& Rheumatology 43(5):995e1000, (2000).

[14] Hunter D.J., Eckstein F., Exercise and osteoarthritis. Journal of Anatomy, 214(2):197e207, (2009)

[15] Moore A.C., Burris D.L., Tribological and material properties for cartilage of and throughout the bovine stifle: support for the altered joint kinematics hypothesis of osteoarthritis. Osteoarthritis Cartilage, 23(1):161e9, (2015).

[16] Zhu F., Wang P., Lee N.H., Goldring M.B., Konstantopoulos K., Prolonged application of high fluid shear to chondrocytes recapitulates gene expression profiles associated with osteoarthritis. PLoS One 5(12):e15174, (2010).

[17] Williams P.T., Effects of running and walking on osteoarthritis and hip replacement risk. Medicine \& Science in Sports \& Exercise, 45(7): 1292e7, (2013).

[18] Moore A.C., Graham B.T., Price C., Burris D.L., Shining a light on the tribological rehydration of cartilage. Tribology \& Lubrication Technology 73 (8), 51, (2017).

[19] Rong M., Liu H., Scaraggi M., Bai Y., Bao L., Ma S., Ma Z., Cai M., Dini D., Zhou F., High Lubricity Meets Load Capacity: Cartilage mimicking bilayer structure by brushing up stiff hydrogels from subsurface, Advanced Functional Materials 30 (39), 2004062, (2020).

[20] Steele JAM,McCullen SD , Callanan A ,Autefage H , Accardi MA ,Dini D , Stevens MM, Combinatorial scaffold morphologies for zonal articular cartilage engineering, Acta Biomaterialia, 10, 5, 2065-2075, (2014).

[21] Rajan V, Caligaris M, Hung CT, Ahmad CS, Ateshian GA. Hemiarthroplasties defeat interstitial fluid pressurization in cartilage and promote greater friction than natural joints. In: Annual Meeting of the Orthopedic Research Society 2010:2120.

[22] De Boera G.N., Raskeb N. , Soltanahmadia S., Dowson D. , Bryant M.G., Hewson R.W.,A porohyperelastic lubrication model for articular cartilage in the natural synovial joint, Tribology International, 149, 105760, (2020).

[23] Liao J.J., Smith D.W., Miramini S. , Gardiner B. S., Zhang L., A coupled contact model of cartilage lubrication in the mixed-mode regime under static compression, Tribology International, 145, 106185 , (2020).

[24] Vanwanseele B., Lucchinetti E., Stüssi E., The effects of immobilization on the characteristics of articular cartilage: current concepts and future directions. Osteoarthritis Cartilage; 10(5):408e19, (2002).

[25] Ekholm R, Ingelmark BE., Functional thickness variations of human articular cartilage. Acta Societatis Medicorum Upsaliensis, 57(1e2): 39e 59, (1951).

[26] Ingelmark BE, Ekholm R., A study on variations in the thickness of articular cartilage in association with rest and periodical load; an experimental investigation on rabbits. Upsala Lakareforen Forh;53(1e2):61e74, (1947).

[27] Accardi M.A., Dini D., Cann P.M., Experimental and numerical investigation of the behaviour of articular cartilage under shear loadingInterstitial fluid pressurisation and lubrication mechanisms. Tribology International, 44(5):565e78, (2011).

[28] Krishnan R., Caligaris M., Mauck R.L., Hung C.T., Costa K.D., Ateshian G.A., Removal of the superficial zone of bovine articular cartilage does not increase its frictional coefficient. Osteoarthritis Cartilage, 12(12):947e55, (2004).

[29] Abaqus Theory Manual, version. Pawtucket, USA: Karlsson Hibbitt,Sorensen, Inc.; (2008)

[30] Abaqus, I, Providence, USA

[31] Wu JZ, Epstein M, Herzog W. Evaluation of the finite element softwareAbaqus for biomechanical modelling of biphasic tissues. Journal of Biomechanics 31(2):165-9, (1997).

[32] Persson B. N. J. Contact mechanics for poroelastic, fluid-filled media, with application to cartilage, The Journal of Chemical Physics 145 (23), 234703, (2016).

[33] Persson B. N. J., Kovalev A. and Gorb S. N. , Simple contact mechanics model of the vertebrate cartilage, Soft Matter, 37, (2017).

[34] Burris D.L., Moore A.C., Cartilage and joint lubrication: new insights into the role of hydrodynamics. Biotribology 12, 8-14, (2017).

[35] Mak A.F., Lai W.M., Mow V.C., Biphasic indentation of articular cartilage - I. Theoretical analysis. Journal of Biomechanics. 20:703-714, (1987)

[36] Mow V.C., Gibbs M.C., Lai W.M., Zhu W.B., Athanasiou K.A., Biphasic indentation of articular cartilage - II. A numerical algorithm and an experimental study. Journal of Biomechanics; 22(8-9):853861, (1989).

[37] Zhang L, Gardiner BS, Smith DW, Pivonka P, Grodzinsky A, A fully coupled poroelastic reactive-transport model of cartilage. Molecular Cellular Biomechanics : MCB,5(2):133-153, (2008).

[38] Miramini, S., Smith, D.W., Zhang, L., and Gardiner, B.S., The spatiotemporal mechanical environment of healthy and injured human cartilage during sustained activity and its role in cartilage damage. Journal of the Mechanical Behavior of Biomedical Materials 74: 1-10, (2017).

[39] Laires P.A., Canhão H., Rodrigues A.M., Eusébio M., Gouveia M., Branco J.C., The impact of osteoarthritis on early exit from work: results from a population-based study, BMC Public Health. 18: 472, (2018). 
[40] Mattei L., Campioni E., Accardi M. A., Dini D., Finite element analysis of the meniscectomised tibio-femoral joint: implementation of advanced articular cartilage models, Computer Methods in Biomechanics and Biomedical Engineering, 17, 14, (2014).

[41] Persson B.N.J., Albohr O., Tartaglino U., Volokitin A. I., Tosatti E. On the nature of surface roughness with application to contact mechanics, sealing, rubber friction and adhesion. Journal of Physics: Condensed Matter, 17, 1, (2004).

[42] Dieker A. B., Mandjes M. On spectral simulation of fractional Brownian motion, Probab. Engrg. Inform. Sci., 17 ,pp. 417-434, ( 2003).

[43] Putignano C., Afferrante L.,Carbone G., Demelio G. A new efficient numerical method for contact mechanics of rough surfaces. International Journal of Solids and Structures, 49 (2), (2012).

[44] Carbone G, Putignano C . Rough viscoelastic sliding contact: theory and experiments, Physical Review E 89 (3), 032408, (2014).

[45] Putignano C, Menga N, Afferrante L, Carbone G .Viscoelasticity induces anisotropy in contacts of rough solids. Journal of the Mechanics and Physics of Solids, 129, 147-159. (2019).

[46] Hamrock B. J., Schmid S.R., Jacobson B. O. Fundamentals of Fluid Film Lubrication, CRC Press, (2004).

[47] Snoeijer J. H., Eggers J., Venner C. H., Similarity theory of lubricated Hertzian contacts, Physics of Fluids, 25, 101705 (2013).

[48] Cunningham R.E., Williams R.J.J., Diffusion in Gases and Porous Media. New York: Plenum Press, (1980).

[49] Bowden F. P., Tabor D., The Friction and Lubrication of Solids, Clarendon Press, (2001).

[50] Patir N. , Cheng H. S. , An Average Flow Model for Determining Effects of Three-Dimensional Roughness on Partial Hydrodynamic Lubrication, Journal of Lubrication Technology, 100(1): 12-17, (1978).

[51] Patir N. , Cheng H. S. , Application of Average Flow Model to Lubrication Between Rough Sliding Surfaces, Journal of Lubrication Technology,101(2): 220-229, (1979).

[52] Sahlin F, Larsson R,Almqvist A, Lugt PM, Marklund P. A mixed lubrication model incorporating measured surface topography.Part1: theory of flow factors. Proceedings Institutions of Mechanical Engineering Part J: Journal of Engineering Tribology, 224:335-51, (2010).

[53] Sahlin F, Larsson R,Almqvist A, Lugt PM, Marklund P. A mixed lubrication model incorporating measured surface topography. Part2: roughness treatment, model validation, and simulation. Proceedings Institutions of Mechanical Engineering Part J: Journal of Engineering Tribology, 224:353-65. (2010)

[54] Scaraggi M, Carbone G, Persson BNJ , Dini D, Lubrication in soft rough contacts: A novel homogenized approach. Part I-Theory, Soft Matter 7 (21), 10395-10406, (2011).

[55] Putignano C, Afferrante L, Carbone G, Demelio GP, A multiscale analysis of elastic contacts and percolation threshold for numerically generated and real rough surfaces, Tribology International 64, 148154, (2013).

[56] Dapp, W.B., Lücke, A., Persson, B.N.J., Müser, M.H. Self-affine elastic contacts: Percolation and leakage, Physical Review Letters, 108 (24), 244301, (2012).

[57] Vlădescu SC, Putignano C , Marx N ,Keppens T, Reddyhoff T , Dini D . The Percolation of Liquid Through a Compliant Seal-An Experimental and Theoretical Study, Journal of Fluids Engineering 141 (3), 031101, (2019).

[58] MathWorks, Matlab User's Guide (R2020a). (2020)

[59] Moore A.C., Burris D.L., Tribological rehydration of cartilage and its potential role in preserving joint health. Osteoarthritis and cartilage 25 (1), 99-107, (2017).

[60] Moore A.C., Schrader J.L., Ulvila J.J., Burris D.L., A review of methods to study hydration effects on cartilage friction TribologyMaterials. Surfaces \& Interfaces, 1-13, (2017).

[61] Linn F.C., .Lubrication of Animal Joints II: The Mechanism. Journal of Biomechanics, 1, 193-205, (1968).

[62] Graham B.T., Moore A.C., Burris D.L., Price C., Mapping the spatiotemporal evolution of solute transport in articular cartilage explants reveals how cartilage recovers fluid within the contact area during sliding, Journal of Biomechanics, 71,271-276, (2018).
[63] Putignano C, Dini D, Soft matter lubrication: does solid viscoelasticity matter?, ACS applied materials \& interfaces 9 (48), 42287-42295, (2017).

[64] Putignano C, Soft lubrication: A generalized numerical methodology. Journal of the Mechanics and Physics of Solids 134, 103748, (2020) 